\title{
Multi-Generations Assessment of Dietary Nucleotides Consumption in Maternal Rats
}

\author{
Xu Meihong ${ }^{1,2}$, Yi Ma Zhao ${ }^{1,2}$, Linlin Xü ${ }^{1,2}$, Yajun Xü ${ }^{1,2}$, Yong Li ${ }^{1,2, *}$ \\ ${ }^{1}$ Department of Nutrition and Food Hygiene, School of Public Health, Peking University, Beijing, PR China \\ ${ }^{2}$ Beijing Key Laboratory of Toxicological Research and Risk Assessment for Food Safety, Peking University, Beijing, PR China \\ *Corresponding author: liyongbmu@163.com
}

Received April 29, 2014; Revised October 20, 2014; Accepted October 26, 2014

\begin{abstract}
This study investigated multi-generational reproductive and developmental toxicity of dietary nucleotides (NTs) during lactation in rats with the purpose of augmenting currently available safety data. Weanling rats (F0) were fed NTs at doses of $0.01 \%, 0.04 \%, 0.16 \%, 0.64 \%$, and $1.28 \%$ (wt/wt for 90 days and then mated in a 1:1 pattern. The F1 generation rats were fed NTs for 90 days after weaning. The process was repeated until F3 rats were born. We measured the physical and neural development landmarks during lactation in the F3 generation and observed the signs of maternal rats during the gestation, parturition, and lactation periods. No significant differences in either physical or neural developmental landmarks were observed between the control and NT groups. No clinically adverse effects were found in the maternal rats at any point in gestation, parturition, or lactation. There were no alterations in the indicators of parturition. No significant differences were observed between controls and NT-treated rats fed four kinds of nucleotides in F0 milk on PND 21. In conclusion, NTs in doses of up to $1.28 \%$ did not cause any adverse effects in either the maternal or filial individuals.
\end{abstract}

Keywords: dietary nucleotides, meternal, safety, Sprague-Dawley rat, multiple generations

Cite This Article: Xu Meihong, Yi Ma Zhao, Linlin Xu, Yajun Xu, and Yong Li, "Multi-Generations Assessment of Dietary Nucleotides Consumption in Maternal Rats.” Journal of Food and Nutrition Research, vol. 2, no. 11 (2014): 800-805. doi: 10.12691/jfnr-2-11-7.

\section{Introduction}

Nucleotides and their metabolic products play key roles in most biological processes. They are basic units of nucleic acids, but they also serve as coenzyme components and biological regulators that transfer chemical energy and act in biosynthetic pathways. Increasing amounts of evidence show that dietary nucleotides affect different aspects of body function and health, including lipid metabolism [1], immune function $[2,3,4,5]$, intestinal microbiota [6], liver function and morphology [7], as well as growth performance [8]. Although nucleotides can be synthesized de novo, several tissues with rapid turnover rates, such as the intestine, may be benefit from an exogenous supply to save energy. Nucleotides become semi-essential nutrients when the body is in a diseased state, a period of limited nutrient intake, rapid growth, and during development [9].

With multifaceted activities, there is a history of safe consumption of NTs in the human diet. Numerous studies have shown that nucleotide-fortified infant formula is safe and beneficial [10-16]. However, most of the NTs-studies have focused on infants or on adults. For instance, few high-level toxic or carcinogenic effects (up to 0.64\%) were observed in Sprague-Dawley rats at any point in their lifetimes in our previous study. Furthermore, our multi-generation assessment of dietary nucleotides (NTs) indicated that may play roles in the promotion of early growth and development after weaning [17]. Nevertheless, whether NTs would be always good for maternal ones?

Here, a multi-generational reproductive toxicity study was conducted with the purpose of augmenting the currently available safety data for NTs consumed in mater throughout their pregnancy, delivery and lactation. The physical and neural developmental landmarks of offspring were recorded to assess toxicity of NTs during lactation. The content of nucleotides in F0 milk was also detected on the $21^{\text {th }}$ day of lactation.

\section{Materials and Methods}

\subsection{Test Substance}

NTs provided by Zhen-Ao Biotechnology Ltd. Co. (Dalian, China) were derived from brew's yeast RNA with purity of more than 99\%. The NTs mixture contained adenosine monophosphate (AMP), guanosine monophosphate (GMP), uridine monophosphate (UMP), and cytidinemonophosphate (CMP) with the following ratio 22.8: 26.6: 30.2: 20.4, respectively. The experimental diet was based on AIN93G formulation [18]. The experimental diet was supplemented with "AIN93G" to give 10, 40, 160, 640, and $1280 \mathrm{mg}$ of mixed $\mathrm{NT} \mathrm{kg}^{-1}$ diet. The food consumption of rats was usually $10 \mathrm{~g} / 100 \mathrm{~g} * \mathrm{bw}$. The dosages were $0.01 \%, 0.04 \%, 0.16 \%, 0.64 \%$ or $1.28 \%$. 
Dietary ingredients (Supplementary_material 1) were thoroughly mixed in a mixer, pelletized, and air-dried at room temperature.

\subsection{Experimental Animal and Housing Conditions}

A total of 360 male and female Sprague-Dawley (S-D) rats (4 weeks old), weighing 35-55 g, were obtained from the Animal Service of Health Science Center, Peking University, Beijing. Rats were housed two per plastic cage with free access to rat chow and tap water in a SPF (specific-pathogen-free) filter-protected air-conditioned room with controlled temperature $\left(25-28^{\circ} \mathrm{C}\right)$, relative humidity (60 $\pm 5 \%)$, and 12-hour light/dark cycles (light on 07:30-19:30 hours). All animals were handled in accordance with the guidelines of the National Institutes of Health and the guidelines of the Peking University Animal Research Committee (www.lab.pku.edu.cn).

\subsection{Experimental Design}

After a 1-week acclimation period, rats were randomly assigned to one of six groups (thirty male and thirty female animals per group): one control group and five experimental groups. Control rats were fed the AIN93G rodent diet (Vital River Ltd. Co., Beijing). The diets given to rats in the five experimental groups were $0.01 \%, 0.04 \%$, $0.16 \%, 0.64 \%$, and $1.28 \%$ NTs, respectively. The experimental diet was supplemented with "AIN93G" to give $10,40,160,640$, and $1280 \mathrm{mg}$ of mixed $\mathrm{NT} \mathrm{kg}^{-1}$ diet. Dietary ingredients were thoroughly mixed in a mixer, pellets were made, and airdried at room temperature. For the mating portion of the multi-generation reproduction, an F0 female was paired with one F0 male from the same treatment group by placing them in the same cage. Vaginal smear examination was used to determine whether female rats were pregnant at 7:00 am the next day. After confirmation of mating, the female was placed in an individual cage, ensuring that 20 female rats in each group had become pregnant. The day of parturition was designated as day 0 of lactation for the dams and postnatal day (PND) 0 for pups (F1). All pups, on PND 4 were examined externally for abnormalities and culled to yield 4 pups/sex/litter. Culled pups were weighed and discarded. The dam and litter remained together and remained on the control or NTs diets until weaning on LD 21. Litters were sexed, examined for gross malformations and the number of stillbirths and live pups were recorded. After weaning, F1 rats were fed the same control or NTs diets as the F0 generation for 90 days. The healthy ones rats in each group were randomly selected to produce the next generation. Multi-generational reproduction was repeated until the F3 generation was born.

\subsection{Maternal Clinical Observations}

Throughout the study, general clinical observations were made each day for mortality and overt signs of toxicity. Behavioral changes, signs of difficult or prolonged parturition and all signs of toxicity were recorded. These included dysphoric disorders, vaginal bleeding, somnolence and mobility-restriction in gestation, spontaneous abortion, dystocia or malformation in parturition, refusal to suckle, biting offspring, nipple swelling, ulceration, and secretion of non-milk substance during lactation.

\subsection{Maternal Body Weight and Feed Intake}

Maternal rats (F0, F1 and F2) were weighed on the first day of dosing and weekly thereafter. Then they were weighed on gestation days $0,7,14$, and 21 and during lactation on the same days as the weighing of litters. These observations were reported individually for each rat. Diet utilization was calculated using the following formula: Diet utilization (\%) = [weight gain (g)/ food consumption (g)] x 100

\subsection{Fetal observations}

The fetal parameters recorded for individual dam included, total number of fetuses, number of abnormal fetuses, number of dead fetuses, number of live fetuses, number of male and female fetuses, weight of fetuses, and sex ratio.

Physical or behavioral abnormalities observed in the dams and offspring were recorded. Live pups were counted and weighed in the same litter at birth (PND 0) and on days 4, 7, 14, and 21 of lactation. Physical development of the offspring (F1, F2, and F3) was recorded mainly in the form of gains in body weight. Other physical parameters were evaluated, including auricular appendages, incisor eruption, and eye opening. Age at testicular descent, vaginal opening, and foreskin separation were also determined. Meanwhile, neural development of the offspring (F1, F2, and F3) was recorded such as forelimb positioning, hindlimb positioning, surface righting, cliff escape, negative gravitropism, and air righting.

\subsection{Assessment of Nucleotides in F0 Milk}

On PND 21, rat pups were removed from the dams more than $2 \mathrm{~h}$ before collection of milk samples. These were collected by manual expression of the mammary gland under $1 \%$ pentobarbital sodium $(0.4 \mathrm{ml} / 100 \mathrm{~g} / \mathrm{bw})$ anesthesia, after intramuscular injection of 1-1.5 U/100 g/bw oxytocin. All samples were stored frozen $\left(-20^{\circ} \mathrm{C}\right)$ until High Performance Liquid Chromatography (HPLC) analysis.

Preparation of milk samples for HPLC analysis was carried out on ice by mixing one volume of milk with one volume of $1.0 \mathrm{M}$ perchloric acid. The mixture was allowed to stand for $10 \mathrm{~min}$ and then centrifuged at 13,400 $\times \mathrm{g}$ for $10 \mathrm{~min}$ at $4^{\circ} \mathrm{C}$. Perchloric acid was extracted from the supernatant by mixing (Vortex) with an equal volume of $0.5 \mathrm{M}$ trioctylamine in 1,1,2-trichlorotrifluoroethane [19]. The incubation mixtures containing milk were chilled and centrifuged in a Microcon 30 concentrator (Amicon Inc., Beverly, MA, U.S.) at $12,000 \times \mathrm{g}$ for 10 min at $4^{\circ} \mathrm{C}$, and the permeate was collected. All samples were filtered through a $0.45 \mu \mathrm{m}$ filter before HPLC analysis.

The analytical determination of PPs was performed by a Varian 9010 reverse phase-HPLC (Walnut Creek, CA, USA). The injector was a Model 7125 Rheodyne manual valve,fitted with a $50 \mu \mathrm{L}$ sample loop. A PerkinElmer (Waltham, MA, USA) LC-235 diode array were connected in series. The system was controlled by a 
PerkinElmer interface module (NCI 900 Network Chromatography Interface). Chromatograms were processed by a PerkinElmer TotalChrom chromatography data handling system. The analytes were separated on a Bondapak C18 reversed phase column (3.9 mm inner diameter $\times 300 \mathrm{~mm}$ length, Waters, Millipore Corp., Milford, MA, USA.) and equipped with an Alltima guard column packed with the same stationary phase.

Samples $(20 \mu \mathrm{L})$ were analyzed. Spectral information was used for peak identification and evaluation of peak purity using Omega-2 and Omega-235 software from Perkin-Elmer. For milk, solvent $\mathrm{A}=0.15 \mathrm{M} \mathrm{KH} 2 \mathrm{PO}$, $\mathrm{pH}=4.00$; solvent $\mathrm{B}=25 \%$ methanol in solvent $\mathrm{A}$. A linear gradient (0-100\% solvent B) started after 2 min of isocratic elution and maintained for $28 \mathrm{~min}$. The flow rate was $1 \mathrm{ml} / \mathrm{min}$ and the detection wavelength was $254 \mathrm{~nm}$.

\subsection{Statistical Analyses}

Statistical analyses were performed using SPSS software (version 19.0, SPSS Inc., Chicago, IL, USA.).
Variances in the measurement data were checked for homogeneity by Bartlett's test. When the data were homogeneous, ANOVA and LSD methods were used. Tamhane's T2 test was used after data are transformed to analyze data among multiple groups if variances were unequal. All reported $P$ values were two-sided. A value of $\mathrm{P}<0.05$ was considered significant.

\section{Results}

\subsection{General Information}

No significant differences were detected between control and NT-treated groups with respect to the incidence of clinical signs of toxicity and regular behaviors in maternal (F0, F1, and F2) rats at any point in the study.

\subsection{Maternal Body Weight and Food Consumption}

Table 1. Weight gain and diet utilization of parental (F0, F1 and F2) rats in gestation

\begin{tabular}{|c|c|c|c|c|c|c|c|c|}
\hline \multirow{2}{*}{ Generation } & \multirow{2}{*}{ Group } & \multirow{2}{*}{ Litter number } & \multicolumn{3}{|c|}{ Weight gain (g) } & \multicolumn{3}{|c|}{ Diet utilization (\%) } \\
\hline & & & Week 1 & Week 2 & Week 3 & Week 1 & Week 2 & Week 3 \\
\hline \multicolumn{9}{|l|}{ F0 } \\
\hline & control & 20 & $27.20 \pm 7.55$ & $28.39 \pm 3.30$ & $95.15 \pm 11.25$ & $5.37 \pm 0.97$ & $5.74 \pm 0.88$ & $1.94 \pm 0.23$ \\
\hline & $0.01 \%$ NTs & 20 & $32.84 \pm 7.42$ & $33.64 \pm 6.35$ & $89.51 \pm 15.05$ & $4.53 \pm 0.78$ & $4.83 \pm 0.72$ & $1.96 \pm 0.24$ \\
\hline & $0.04 \%$ NTs & 20 & $29.84 \pm 10.52$ & $29.45 \pm 6.89$ & $80.62 \pm 27.69$ & $4.95 \pm 1.10$ & $5.28 \pm 1.44$ & $2.26 \pm 1.04$ \\
\hline & $0.16 \%$ NTs & 20 & $31.26 \pm 8.70$ & $27.32 \pm 10.22$ & $83.8 \pm 29.75$ & $5.01 \pm 1.30$ & $8.56 \pm 10.89$ & $3.98 \pm 7.34$ \\
\hline & $0.64 \%$ NTs & 20 & $32.73 \pm 10.52$ & $29.39 \pm 9.98$ & $87.3 \pm 30.29$ & $5.05 \pm 1.27$ & $9.83 \pm 17.26$ & $4.30 \pm 7.99$ \\
\hline & $1.28 \% \mathrm{NTs}$ & 20 & $35.59 \pm 5.35$ & $30.01 \pm 8.93$ & $84.05 \pm 18.33$ & $4.38 \pm 0.75$ & $6.18 \pm 2.10$ & $2.22 \pm 0.63$ \\
\hline \multicolumn{9}{|l|}{ F1 } \\
\hline & control & 20 & $32.10 \pm 20.93$ & $28.90 \pm 14.33$ & $62.59 \pm 38.19$ & $5.73 \pm 4.71$ & $9.30 \pm 7.51$ & $4.60 \pm 4.96$ \\
\hline & $0.01 \%$ NTs & 20 & $36.79 \pm 9.53$ & $33.55 \pm 5.25$ & $95.34 \pm 14.81$ & $4.60 \pm 1.46$ & $5.29 \pm 0.96$ & $2.05 \pm 0.35$ \\
\hline & $0.04 \%$ NTs & 20 & $38.08 \pm 9.57$ & $30.51 \pm 8.22$ & $67.96 \pm 40.50$ & $4.01 \pm 0.86$ & $6.03 \pm 1.81$ & $1.53 \pm 2.72$ \\
\hline & $0.16 \% \mathrm{NTs}$ & 20 & $31.79 \pm 10.80$ & $28.83 \pm 10.39$ & $70.89 \pm 36.05$ & $5.31 \pm 1.85$ & $6.61 \pm 2.63$ & $2.55 \pm 0.74$ \\
\hline & $0.64 \%$ NTs & 20 & $34.46 \pm 26.42$ & $24.88 \pm 21.23$ & $70.63 \pm 41.67$ & $3.49 \pm 3.50$ & $6.28 \pm 10.19$ & $2.12 \pm 0.42$ \\
\hline & $1.28 \% \mathrm{NTs}$ & 20 & $28.38 \pm 15.35$ & $22.18 \pm 13.64$ & $68.93 \pm 50.02$ & $2.10 \pm 7.14$ & $8.89 \pm 6.30$ & $3.51 \pm 4.15$ \\
\hline \multicolumn{9}{|l|}{ F2 } \\
\hline & Control & 20 & $28.29 \pm 8.55$ & $27.50 \pm 5.60$ & $90.55 \pm 19.97$ & $5.69 \pm 1.86$ & $6.03 \pm 0.78$ & $2.15 \pm 0.70$ \\
\hline & $0.01 \% \mathrm{NTs}$ & 20 & $36.47 \pm 7.74$ & $29.38 \pm 9.46$ & $88.44 \pm 61.20$ & $4.59 \pm 1.01$ & $5.06 \pm 0.86$ & $2.49 \pm 2.83$ \\
\hline & $0.04 \% \mathrm{NTs}$ & 20 & $28.84 \pm 6.15$ & $30.24 \pm 9.07$ & $88.58 \pm 17.61$ & $5.95 \pm 2.08$ & $8.03 \pm 8.18$ & $2.33 \pm 0.82$ \\
\hline & $0.16 \%$ NTs & 20 & $22.08 \pm 7.73$ & $26.67 \pm 6.51$ & $90.32 \pm 20.22$ & $5.17 \pm 2.68$ & $5.12 \pm 0.79$ & $2.33 \pm 0.59$ \\
\hline & $0.64 \%$ NTs & 20 & $29.21 \pm 6.64$ & $32.72 \pm 9.96$ & $99.37 \pm 16.84$ & $4.18 \pm 0.63$ & $5.75 \pm 0.84$ & $2.06 \pm 0.21$ \\
\hline & $1.28 \% \mathrm{NTs}$ & 20 & $34.24 \pm 6.83$ & $25.69 \pm 6.44$ & $105.45 \pm 14.71$ & $4.59 \pm 0.91$ & $4.90 \pm 0.49$ & $1.95 \pm 0.23$ \\
\hline
\end{tabular}

Data are mean \pm .SD values.

Table 2. Weight gain and diet utilization of maternal (F0, F1 and F2) rats in lactation

\begin{tabular}{|c|c|c|c|c|c|c|c|c|}
\hline \multirow{2}{*}{ Generation } & \multirow{2}{*}{ Group } & \multirow{2}{*}{ Litter number } & \multicolumn{3}{|c|}{ Weight gain (g) } & \multicolumn{3}{|c|}{ Diet utilization (\%) } \\
\hline & & & Week 1 & Week 2 & Week 3 & Week 1 & Week 2 & Week 3 \\
\hline \multicolumn{9}{|l|}{ F0 } \\
\hline & control & 20 & $-4.99 \pm 12.28$ & $-3.09 \pm 13.90$ & $-17.69 \pm 13.01$ & $-45.98 \pm 143.76$ & $-47.34 \pm 429.00$ & $-59.83 \pm 160.80$ \\
\hline & $0.01 \% \mathrm{NTs}$ & 20 & $-8.14 \pm 18.55$ & $2.63 \pm 15.14$ & $-21.86 \pm 9.29$ & $23.98 \pm 93.23$ & $-29.06 \pm 78.04$ & $-14.54 \pm 21.59$ \\
\hline & $0.04 \%$ NTs & 20 & $-11.18 \pm 19.57$ & $1.59 \pm 8.66$ & $-12.15 \pm 15.60$ & $-8.69 \pm 31.30$ & $35.08 \pm 176.85$ & $-5.50 \pm 67.37$ \\
\hline & $0.16 \% \mathrm{NTs}$ & 20 & $-1.07 \pm 7.60$ & $-1.93 \pm 11.49$ & $-15.89 \pm 13.26$ & $-0.18 \pm 41.43$ & $65.50 \pm 141.42$ & $-29.52 \pm 33.68$ \\
\hline & $0.64 \%$ NTs & 20 & $-11.92 \pm 14.15$ & $1.17 \pm 12.50$ & $-17.66 \pm 11.00$ & $-10.51 \pm 29.63$ & $-28.79 \pm 154.5$ & $-40.98 \pm 37.58$ \\
\hline & $1.28 \% \mathrm{NTs}$ & 20 & $-5.38 \pm 11.96$ & $-0.60 \pm 14.39$ & $-15.44 \pm 12.30$ & $9.28 \pm 87.08$ & $30.05 \pm 134.82$ & $-26.48 \pm 78.61$ \\
\hline \multicolumn{9}{|l|}{ F1 } \\
\hline & control & 20 & $-6.90 \pm 18.97$ & $12.88 \pm 19.02$ & $-10.98 \pm 20.12$ & $-21.56 \pm 30.06$ & $41.13 \pm 50.09$ & $-0.44 \pm 79.83$ \\
\hline & $0.01 \% \mathrm{NTs}$ & 20 & $-1.99 \pm 13.99$ & $11.48 \pm 7.56$ & $-29.58 \pm 29.09$ & $1.38 \pm 45.06$ & $48.67 \pm 41.67$ & $-11.38 \pm 17.34$ \\
\hline & $0.04 \% \mathrm{NTs}$ & 20 & $1.05 \pm 13.35$ & $14.33 \pm 13.16$ & $-29.33 \pm 34.90$ & $15.36 \pm 16.62$ & $50.59 \pm 31.84$ & $-22.08 \pm 16.60$ \\
\hline & $0.16 \%$ NTs & 20 & $3.64 \pm 10.10$ & $5.41 \pm 22.99$ & $-14.50 \pm 27.45$ & $-3.01 \pm 32.75$ & $2.74 \pm 37.92$ & $24.06 \pm 64.88$ \\
\hline & $0.64 \% \mathrm{NTs}$ & 20 & $-1.58 \pm 18.17$ & $-6.22 \pm 19.49$ & $-11.13 \pm 21.41$ & $-0.82 \pm 35.25$ & $50.89 \pm 152.85$ & $-31.05 \pm 75.89$ \\
\hline & $1.28 \%$ NTs & 20 & $13.80 \pm 28.62$ & $8.69 \pm 16.25$ & $-6.11 \pm 24.32$ & $5.87 \pm 18.93$ & $27.74 \pm 85.22$ & $8.94 \pm 52.02$ \\
\hline \multicolumn{9}{|l|}{ F2 } \\
\hline & Control & 20 & $-10.73 \pm 26.63$ & $13.74 \pm 19.08$ & $-11.62 \pm 19.71$ & $-10.71 \pm 8.07$ & $21.39 \pm 15.95$ & $-23.66 \pm 18.42$ \\
\hline & $0.01 \% \mathrm{NTs}$ & 20 & $-15.89 \pm 21.62$ & $9.87 \pm 23.63$ & $-11.89 \pm 21.81$ & $-12.43 \pm 12.94$ & $4.28 \pm 52.33$ & $-12.70 \pm 25.80$ \\
\hline & $0.04 \%$ NTs & 20 & $-18.12 \pm 17.68$ & $10.15 \pm 9.69$ & $-8.82 \pm 13.27$ & $-11.91 \pm 21.83$ & $27.11 \pm 24.46$ & $-15.00 \pm 43.87$ \\
\hline & $0.16 \%$ NTs & 20 & $-13.51 \pm 16.11$ & $6.67 \pm 15.73$ & $-11.02 \pm 12.86$ & $-8.63 \pm 50.19$ & $16.45 \pm 26.00$ & $-41.78 \pm 23.96$ \\
\hline & $0.64 \%$ NTs & 20 & $-20.41 \pm 14.75$ & $8.49 \pm 11.86$ & $-15.04 \pm 17.75$ & $-8.80 \pm 9.47$ & $28.58 \pm 38.37$ & $-12.58 \pm 43.46$ \\
\hline & $1.28 \%$ NTs & 20 & $-15.76 \pm 14.88$ & $16.80 \pm 11.97$ & $-22.20 \pm 11.88$ & $-7.95 \pm 21.93$ & $18.89 \pm 6.76$ & $-26.64 \pm 20.39$ \\
\hline
\end{tabular}

Data are mean. \pm SD values. 
During the gestation (Table 1) and lactation (Table 2) periods, the weekly weight gain and diet utilization of maternal rats (F0, F1, and F2) did not show any significant differences between the NTs-treated rats and control rats $(\mathrm{P}>0.05)$.

\subsection{Fetal Observations}

Table 3. The parturition data of parental (F0, F1 and F2) rats

\begin{tabular}{|c|c|c|c|c|c|c|c|c|}
\hline Generations & Group & $\begin{array}{c}\text { Litter } \\
\text { number }\end{array}$ & $\begin{array}{c}\text { Average number of } \\
\text { living fetuses per } \\
\text { litter }\end{array}$ & $\begin{array}{c}\text { Average weight } \\
\text { per litter (g) }\end{array}$ & $\begin{array}{c}\text { Number of } \\
\text { dead fetuses }\end{array}$ & $\begin{array}{c}\text { Number of } \\
\text { fetuses with } \\
\text { birth defect }\end{array}$ & $\begin{array}{c}\text { Average body } \\
\text { weight (g) }\end{array}$ & $\begin{array}{c}\text { Sex ratio } \\
\text { (male/female) }\end{array}$ \\
\hline F0 & & & & & & & & \\
\hline & control & 20 & $13.83 \pm 1.89$ & $88.31 \pm 11.82$ & 2 & 1 & $6.40 \pm 0.51$ & $1.27 \pm 1.26$ \\
\hline & 0.01 & 20 & $14.33 \pm 2.17$ & $92.06 \pm 16.44$ & 2 & 0 & $6.43 \pm 0.69$ & $1.65 \pm 2.65$ \\
\hline & 0.04 & 20 & $12.65 \pm 4.34$ & $77.85 \pm 24.99$ & 1 & 0 & $6.35 \pm 0.82$ & $1.31 \pm 1.00$ \\
\hline & 0.16 & 20 & $12.93 \pm 3.50$ & $81.38 \pm 22.11$ & 1 & 0 & $6.34 \pm 0.76$ & $1.15 \pm 0.97$ \\
\hline & 0.64 & 20 & $14.39 \pm 1.65$ & $89.53 \pm 9.67$ & 1 & 0 & $6.25 \pm 0.48$ & $1.17 \pm 0.49$ \\
\hline & 1.28 & 20 & $12.44 \pm 3.28$ & $82.24 \pm 19.35$ & 0 & 0 & $6.75 \pm 0.90$ & $1.08 \pm 0.71$ \\
\hline F1 & & & & & & & & \\
\hline & control & 20 & $13.88 \pm 3.00$ & $86.13 \pm 13.63$ & 2 & 0 & $6.22 \pm 0.58$ & $1.40 \pm 0.67$ \\
\hline & 0.01 & 20 & $14.30 \pm 3.06$ & $94.21 \pm 13.95$ & 1 & 0 & $6.63 \pm 0.64$ & $1.32 \pm 0.78$ \\
\hline & 0.04 & 20 & $12.38 \pm 4.00$ & $79.78 \pm 24.73$ & 0 & 0 & $6.50 \pm 0.43$ & $1.29 \pm 0.74$ \\
\hline & 0.16 & 20 & $12.43 \pm 3.46$ & $80.83 \pm 18.02$ & 0 & 0 & $6.63 \pm 0.75$ & $1.16 \pm 0.57$ \\
\hline & 0.64 & 20 & $13.67 \pm 3.50$ & $87.42 \pm 24.64$ & 1 & 0 & $6.29 \pm 0.64$ & $1.11 \pm 0.62$ \\
\hline & 1.28 & 20 & $14.86 \pm 1.46$ & $95.00 \pm 7.14$ & 1 & 0 & $6.36 \pm 0.41$ & $1.10 \pm 0.59$ \\
\hline F2 & & & & & & & & \\
\hline & control & 20 & $14.15 \pm 2.41$ & $89.93 \pm 15.14$ & 1 & 0 & $6.30 \pm 0.40$ & $1.05 \pm 0.80$ \\
\hline & 0.01 & 20 & $14.40 \pm 2.47$ & $97.99 \pm 15.77$ & 2 & 0 & $6.69 \pm 0.76$ & $1.20 \pm 0.62$ \\
\hline & 0.04 & 20 & $12.64 \pm 3.10$ & $79.30 \pm 18.54$ & 0 & 0 & $6.31 \pm 0.53$ & $0.96 \pm 0.65$ \\
\hline & 0.16 & 20 & $11.91 \pm 3.39$ & $79.15 \pm 22.26$ & 2 & 0 & $7.37 \pm 3.32$ & $1.66 \pm 1.01$ \\
\hline & 0.64 & 20 & $14.27 \pm 1.83$ & $92.85 \pm 10.95$ & 1 & 0 & $7.67 \pm 4.98$ & $0.90 \pm 0.42$ \\
\hline & 1.28 & 20 & $13.46 \pm 1.45$ & $87.28 \pm 8.71$ & 1 & 0 & $6.34 \pm 0.81$ & $1.26 \pm 0.79$ \\
\hline
\end{tabular}

Data are mean \pm .SD values.

Compared with the control, in lactation, there were no remarkable differences on weight gain and diet utilization in NTs-treated filial rats (F1, F2, and F3) $(\mathrm{P}>0.05)$ (data not shown for simple).
The parturition data of filial (F1, F2, and F3) rats (including average weight, number of living fetuses per litter, average body weight, and sex ratio) are summarized in Table 3. There were no statistically significant differences in the NTs group relative to the control $(\mathrm{P}>0.05)$.
As shown in Tables 4 and 5, the time required for compliance of physical and neural development landmarks did not differ among the groups across multiple generations $(\mathrm{P}>0.05)$.

Table 4. Compliance time of physiological development indicators in F1, F2 and F3 rats

\begin{tabular}{|c|c|c|c|c|c|c|c|c|}
\hline Generation & Group & $\begin{array}{c}\text { Litter } \\
\text { number }\end{array}$ & $\begin{array}{c}\text { Pinna } \\
\text { detachment (d) }\end{array}$ & $\begin{array}{c}\text { Incisor } \\
\text { eruption (d) }\end{array}$ & $\begin{array}{c}\text { Eyes } \\
\text { opening (d) }\end{array}$ & $\begin{array}{c}\text { Vaginal } \\
\text { opening (d) }\end{array}$ & $\begin{array}{c}\text { Testicular } \\
\text { descent (d) }\end{array}$ & $\begin{array}{c}\text { Foreskin } \\
\text { separation (d) }\end{array}$ \\
\hline F0 & & & & & & & \\
\hline & control & 20 & $3.19 \pm 0.57$ & $11.92 \pm 0.69$ & $14.31 \pm 0.60$ & $34.89 \pm 1.49$ & $21.86 \pm 0.46$ & $43.39 \pm 1.71$ \\
\hline & $0.01 \% N T s$ & 20 & $3.00 \pm 0.47$ & $11.39 \pm 0.64$ & $13.84 \pm 0.60$ & $33.74 \pm 1.52$ & $21.70 \pm 0.42$ & $42.17 \pm 2.08$ \\
\hline & $0.04 \%$ NTs & 20 & $3.18 \pm 0.72$ & $11.29 \pm 0.99$ & $14.32 \pm 0.93$ & $34.06 \pm 1.72$ & $21.56 \pm 0.42$ & $42.94 \pm 1.83$ \\
\hline & $0.16 \%$ NTs & 20 & $3.00 \pm 0.71$ & $11.61 \pm 0.98$ & $14.04 \pm 0.57$ & $33.77 \pm 1.45$ & $21.44 \pm 0.17$ & $42.78 \pm 1.48$ \\
\hline & $0.64 \%$ NTs & 20 & $3.44 \pm 0.62$ & $11.25 \pm 0.77$ & $14.14 \pm 0.61$ & $33.56 \pm 1.75$ & $21.50 \pm 0.00$ & $42.54 \pm 1.92$ \\
\hline & $1.28 \% N T s$ & 20 & $3.06 \pm 0.54$ & $11.31 \pm 1.00$ & $13.62 \pm 0.55$ & $33.15 \pm 1.10$ & $21.54 \pm 0.35$ & $42.71 \pm 2.32$ \\
\hline F1 & & & & & & & & \\
\hline & control & 20 & $3.25 \pm 0.21$ & $11.31 \pm 1.13$ & $14.81 \pm 0.59$ & $34.44 \pm 0.56$ & $21.38 \pm 0.69$ & $45.25 \pm 2.76$ \\
\hline & $0.01 \% N T s$ & 20 & $2.94 \pm 0.19$ & $10.83 \pm 0.71$ & $14.33 \pm 0.66$ & $33.94 \pm 0.53$ & $21.28 \pm 0.71$ & $46.56 \pm 2.83$ \\
\hline & $0.04 \% N T s$ & 20 & $2.75 \pm 0.21$ & $10.63 \pm 0.52$ & $14.13 \pm 0.74$ & $33.81 \pm 0.56$ & $21.44 \pm 0.62$ & $46.43 \pm 2.49$ \\
\hline & $0.16 \% N T s$ & 20 & $3.43 \pm 0.22$ & $11.71 \pm 0.91$ & $14.86 \pm 0.94$ & $33.64 \pm 0.60$ & $21.93 \pm 0.98$ & $46.07 \pm 3.00$ \\
\hline & $0.64 \% N T s$ & 20 & $2.61 \pm 0.19$ & $11.33 \pm 0.87$ & $14.06 \pm 1.07$ & $33.00 \pm 0.56$ & $21.75 \pm 0.71$ & $43.75 \pm 1.83$ \\
\hline & $1.28 \% N T s$ & 20 & $2.71 \pm 0.22$ & $10.93 \pm 1.13$ & $14.07 \pm 0.73$ & $34.00 \pm 0.60$ & $21.29 \pm 0.81$ & $43.64 \pm 2.85$ \\
\hline & & & & & & & & \\
\hline & Control & 20 & $2.88 \pm 0.38$ & $11.42 \pm 0.90$ & $14.42 \pm 0.36$ & $35.39 \pm 0.45$ & $19.64 \pm 0.24$ & $42.86 \pm 1.03$ \\
\hline & $0.01 \% N T s$ & 20 & $2.80 \pm 0.56$ & $11.30 \pm 0.80$ & $14.23 \pm 0.53$ & $35.00 \pm 0.43$ & $19.61 \pm 0.21$ & $43.20 \pm 1.75$ \\
\hline & $0.04 \% N T s$ & 20 & $2.82 \pm 0.42$ & $11.15 \pm 0.69$ & $14.00 \pm 0.46$ & $34.78 \pm 0.45$ & $19.96 \pm 0.24$ & $42.95 \pm 1.74$ \\
\hline & $0.16 \% N T s$ & 20 & $2.80 \pm 0.54$ & $11.30 \pm 0.63$ & $14.55 \pm 0.44$ & $34.78 \pm 0.45$ & $20.25 \pm 0.25$ & $43.67 \pm 1.25$ \\
\hline & $0.64 \% N T s$ & 20 & $2.87 \pm 0.52$ & $11.43 \pm 0.59$ & $14.10 \pm 0.66$ & $35.60 \pm 0.43$ & $20.18 \pm 0.21$ & $42.60 \pm 1.51$ \\
\hline & $1.28 \% N T s$ & 20 & $2.89 \pm 0.42$ & $11.23 \pm 0.44$ & $14.31 \pm 0.78$ & $35.15 \pm 0.43$ & $20.05 \pm 0.24$ & $42.25 \pm 1.21$ \\
\hline
\end{tabular}


Table 5. Compliance time of neural developmental landmarks in filial (F1, F2 and F3) rats

\begin{tabular}{|c|c|c|c|c|c|c|c|c|}
\hline Generation & Group & $\begin{array}{c}\text { Litter } \\
\text { number }\end{array}$ & $\begin{array}{c}\text { Forelimb } \\
\text { positioning (d) }\end{array}$ & $\begin{array}{c}\text { Hindlimb } \\
\text { positioning (d) }\end{array}$ & $\begin{array}{c}\text { Surface } \\
\text { righting (d) }\end{array}$ & $\begin{array}{l}\text { Cliff escape } \\
\text { (d) }\end{array}$ & $\begin{array}{c}\text { Slope } \\
\text { turning(d) }\end{array}$ & $\begin{array}{l}\text { Air righting } \\
\text { (d) }\end{array}$ \\
\hline \multicolumn{9}{|l|}{ F0 } \\
\hline & control & 20 & $3.86 \pm 0.56$ & $4.42 \pm 1.33$ & $4.81 \pm 1.24$ & $7.06 \pm 1.37$ & $7.53 \pm 1.36$ & $14.29 \pm 0.75$ \\
\hline & $0.01 \%$ NTs & 20 & $3.87 \pm 0.83$ & $4.42 \pm 1.25$ & $4.29 \pm 0.69$ & $6.92 \pm 1.60$ & $7.34 \pm 1.74$ & $13.79 \pm 0.77$ \\
\hline & $0.04 \%$ NTs & 20 & $3.88 \pm 0.57$ & $4.35 \pm 1.33$ & $4.74 \pm 0.97$ & $7.44 \pm 1.21$ & $7.88 \pm 1.48$ & $13.77 \pm 0.69$ \\
\hline & $0.16 \%$ NTs & 20 & $4.07 \pm 0.62$ & $4.54 \pm 1.29$ & $4.61 \pm 0.90$ & $6.86 \pm 0.84$ & $6.86 \pm 1.05$ & $13.86 \pm 1.03$ \\
\hline & $0.64 \%$ NTs & 20 & $4.06 \pm 0.80$ & $4.67 \pm 1.41$ & $4.92 \pm 1.33$ & $6.89 \pm 1.43$ & $7.62 \pm 1.28$ & $14.19 \pm 0.55$ \\
\hline & $1.28 \% \mathrm{NTs}$ & 20 & $3.92 \pm 0.62$ & $4.22 \pm 1.25$ & $4.36 \pm 0.78$ & $6.22 \pm 1.20$ & $6.75 \pm 1.31$ & $14.03 \pm 0.70$ \\
\hline \multicolumn{9}{|l|}{ F1 } \\
\hline & control & 20 & $3.68 \pm 0.52$ & $4.42 \pm 1.33$ & $7.38 \pm 0.99$ & $7.31 \pm 0.27$ & $7.00 \pm 0.80$ & $13.94 \pm 1.40$ \\
\hline & $0.01 \% N T s$ & 20 & $3.87 \pm 0.69$ & $4.42 \pm 1.25$ & $7.94 \pm 1.70$ & $7.06 \pm 0.26$ & $6.94 \pm 0.46$ & $13.00 \pm 1.32$ \\
\hline & $0.04 \%$ NTs & 20 & $3.89 \pm 0.57$ & $4.35 \pm 1.33$ & $6.88 \pm 0.99$ & $6.94 \pm 0.27$ & $6.63 \pm 0.88$ & $12.75 \pm 1.41$ \\
\hline & $0.16 \%$ NTs & 20 & $3.95 \pm 0.60$ & $4.54 \pm 1.29$ & $9.07 \pm 1.61$ & $7.93 \pm 0.29$ & $7.43 \pm 1.27$ & $13.86 \pm 1.28$ \\
\hline & $0.64 \%$ NTs & 20 & $3.67 \pm 0.45$ & $4.67 \pm 1.41$ & $7.11 \pm 1.02$ & $7.17 \pm 0.26$ & $7.11 \pm 0.33$ & $13.33 \pm 0.56$ \\
\hline & $1.28 \% \mathrm{NTs}$ & 20 & $3.88 \pm 0.65$ & $4.22 \pm 1.25$ & $7.43 \pm 1.05$ & $7.50 \pm 0.29$ & $7.07 \pm 0.61$ & $13.00 \pm 1.04$ \\
\hline \multicolumn{9}{|l|}{ F2 } \\
\hline & Control & 20 & $3.31 \pm 0.25$ & $3.69 \pm 0.43$ & $7.00 \pm 0.89$ & $6.50 \pm 0.54$ & $6.50 \pm 0.46$ & $12.33 \pm 0.81$ \\
\hline & $0.01 \%$ NTs & 20 & $3.27 \pm 0.26$ & $3.57 \pm 0.50$ & $7.07 \pm 0.80$ & $6.67 \pm 0.49$ & $6.57 \pm 0.50$ & $11.97 \pm 0.69$ \\
\hline & $0.04 \%$ NTs & 20 & $3.21 \pm 0.26$ & $3.64 \pm 0.53$ & $6.54 \pm 0.48$ & $6.62 \pm 0.36$ & $6.54 \pm 0.43$ & $12.15 \pm 0.80$ \\
\hline & $0.16 \%$ NTs & 20 & $3.15 \pm 0.24$ & $3.50 \pm 0.33$ & $6.90 \pm 0.61$ & $6.55 \pm 0.50$ & $6.55 \pm 0.60$ & $12.25 \pm 0.72$ \\
\hline & $0.64 \%$ NTs & 20 & $3.10 \pm 0.21$ & $3.43 \pm 0.37$ & $6.77 \pm 0.82$ & $6.77 \pm 0.42$ & $6.57 \pm 0.32$ & $11.90 \pm 0.74$ \\
\hline & $1.28 \% N T s$ & 20 & $3.15 \pm 0.24$ & $3.31 \pm 0.38$ & $6.50 \pm 0.50$ & $6.73 \pm 0.39$ & $6.77 \pm 0.33$ & $11.81 \pm 0.33$ \\
\hline
\end{tabular}

\subsection{Detection of Nucleotides in Fo Milk}

For the content of nucleotides in F0 milk, as shown in Table 6, there was no dramatic difference between the control and experimental NT groups $(\mathrm{P}>0.05)$.

Table 6. Nucleotide levels in milk form F0 rats treated with different dose of dietary nucleotides

\begin{tabular}{ccccc}
\hline Group & $\mathrm{N}$ & AMP $(\mu \mathrm{g} / \mathrm{ml})$ & GMP $(\mu \mathrm{g} / \mathrm{ml})$ & $\mathrm{UMP}(\mu \mathrm{g} / \mathrm{ml})$ \\
\hline control & 4 & $34.11 \pm 18.63$ & $39.98 \pm 11.39$ & $26.00 \pm 9.15$ \\
$0.01 \%$ NTs & 5 & $50.57 \pm 26.68$ & $44.43 \pm 21.52$ & $22.12 \pm 5.79$ \\
$0.04 \%$ NTs & 5 & $34.51 \pm 25.79$ & $49.72 \pm 36.98$ & $10.40 \pm 15.87$ \\
$0.16 \%$ NTs & 4 & $50.47 \pm 27.87$ & $48.83 \pm 8.04$ & $11.54 \pm 10.38$ \\
$0.64 \%$ NTs & 4 & $19.94 \pm 7.68$ & $36.01 \pm 12.27$ & $6.70 \pm 8.47$ \\
$1.28 \%$ NTs & 4 & $28.22 \pm 12.88$ & $54.04 \pm 31.04$ & $13.79 \pm 13.38$ \\
\hline
\end{tabular}

Adenosine monophosphate (AMP), guanosine monophosphate (GMP), uridine monophosphate (UMP) and cytidinemonophosphate (CMP) Data are mean. $\pm S D$ values.

\section{Discussion}

NTs, identified as conditionally essential nutrients, have gained increasing popularity in the diet because they seem to play an important role in human nutrition at different stages of life. Infant formulas and health food supplemented with monophosphate nucleotides are already on both the American and European markets. This study evaluated the safety of NT supplementation for maternal ones with the purpose of augmenting currently available safety data across multiple generations.

Rats are often used as models in assessments of potential reproductive and cumulative dietary risks to humans. Results showed that nucleic acid oral solutions had non mutagenic effects on somatic and germ cells $[20,21]$.

In this study, no clinical adverse effects (including weight gain, food consumption, and signs of toxicity) were observed in the maternal rats at any point in gestation, parturition, or lactation. There were no alterations in the indicators of parturition, such as average weight per litter, number of living fetuses, average body weight, and sex ratio. Then we analyzed the content of four kinds of nucleotides in F0 milk on PND 21. No significant differences from the control were noted in NTs-treated groups. For the filial part, there were no significant differences in physical or neural development landmarks between the control and NT groups. However, in previous study [17,22], NTs consumption could promote growth and development in weaned rats. It has been suggested that NTs could reduce the incidence of diarrhea, possibly by a favorable effect on the gastrointestinal microbiota, in formula-fed infants. In present study revealed that the content of four kinds of nucleotides was not changed by NTs supplementation, in rats' milk on PND 21. It was suggested the nucleotides level was higher in the high-dose rats, which may be due to reproductive function accumulation and differentiation.

In addition, it can be seen in the study that NTs have no adverse effects at up to $1.28 \%$. This is consistent with the data from multi-generational study of development [17]. The dose of up to $1.28 \%$ NTs is the equivalent of 76.8 (g/day/person). In this way, the data presented would provide useful information to assess the risk to human health for NTs.

This experimental design has one drawback that must be noted. We assessed only four kinds of nucleotides in F0 milk and only on PND 21. The composition and concentration of most nucleotides changes during lactation. In addition, we did not record the copulation index. All of these points should be included in further studies.

\section{Conclusions}

In conclusion, a multigeneration assessment of NTs is reported in mater throughout their pregnancy, delivery and lactation and offspring of lactation, in which it was safe even at high dose supplementation. Moreover, the physical and neural developmental landmarks of offspring 
were shown non-toxicity of NTs during lactation. The NOAEL is considered to be $1.28 \%$.

\section{Acknowledgement}

The authors are grateful to Zhen-Ao Biotechnology Ltd. for providing the samples of dietary nucleotides used in this study.

\section{References}

[1] Sanchez PA, Gil A. Nucleotides as semiessential nutritional components. Br J Nutr. 2002; 87 (Suppl 1): S135-7.

[2] Arnaud A, López-Pedrosa JM, Torres MI, Gil A. Dietary nucleotides modulate mitochondrial function of intestinal mucosa in weanling rats with chronic diarrhea. J Pediatr Gastroenterol Nutr. 2003; 37 (2): 124-31.

[3] Frankic T, Pajk T, Rezar V, Levart A, Salobir J. The role of dietary nucleotides in reduction of DNA damage induced by T-2 toxin and deoxynivalenol in chicken leukocytes. Food Chem Toxicol. 2006; 44 (11): 1838-44.

[4] Fontana L, Moreira E, Torres MI, Fernández I, Ríos A, Sánchez de Medina F, Gil A. Dietary nucleotides correct plasma and liver microsomal fatty acid alterations in rats with liver cirrhosis induced by oral intake of thioacetamide. J Hepatol. 1998; 28 (4): 662-9.

[5] Tahmasebi KA, Keyvanshokooh S, Nematollahi A, Mahmoudi N, Pasha-Zanoosi H. Dietary administration of nucleotides to enhance growth, humoral immune responses, and disease resistance of the rainbow trout (Oncorhynchus mykiss) fingerlings. Fish Shellfish Immunol. 2011; 30 (1): 189-93.

[6] Ortega A, Gil A, Sanchez-Pozo A. Exogenous nucleosides modulate expression and activity of transcription factors in Caco-2 cells. J Nutr Biochem. 2011; 22 (6): 595-604.

[7] Lopez-Navarro AT, Ortega MA, Peragon J, Gil A, Sánchez-Pozo A. Deprivation of dietary nucleotides decreases protein synthesis in the liver and small intestine in rats. Gastroenterology. 1996; 110 (6): 1760-9.

[8] Yu VY, 1998. The role of dietary nucleotides in neonatal and infant nutrition. Singapore Med J. 1998; 39 (4): 145-50.

[9] Singhal A, Kennedy K, Lanigan J, Clough H, Jenkins W, EliasJones A, Stephenson T, Dudek P, Lucas A. Dietary nucleotides and early growth in formula-fed infants: a randomized controlled trial. Pediatrics. 2010; 126 (4): e946-53.

[10] Aggett P, Leach JL, Rueda R, et al. Innovation in infant formula development: A reassessment of ribonucleotides in 2002 . Nutrition. 2003; 19 (4): 375-84.

[11] Boza J. Nucleotides in infant nutrition. Monatsschrift fur Kinderheilkunde. 1998; 146 (supp 1): S39-S48.

[12] Maldonado J, Navarro J, Narbona E, Gil A. The influence of dietary nucleotides on humoral and cell immunity in the neonate and lactating infant. Early Hum Dev. 2001; 65 (Suppl): S69-74.

[13] Sauer N, Bauer E, Vahjen W, et al. Nucleotides modify growth of selected intestinal bacteria in vitro. Livestock Science. 2010; 133: 161-163.

[14] Singhal A, Macfarlane G, Macfarlane S, Lanigan J, Kennedy K, Elias-Jones A, Stephenson T, Dudek P, Lucas A. Dietary nucleotides and fecal microbiota in formula-fed infants: a randomized controlled trial. Am J Clin Nutr. 2008; 87 (6): 178592.

[15] Yu VY. Scientific rationale and benefits of nucleotide supplementation of infant formula. J Paediatr Child Health. 2002; 38 (6): 543-9.

[16] Koletzko B, Baker S, Cleghorn G, Neto UF, Gopalan S, Hernell O, Hock QS, Jirapinyo P, Lonnerdal B, Pencharz P, Pzyrembel H, Ramirez-Mayans J, Shamir R, Turck D, Yamashiro Y, Zong-Yi D. Global standard for the composition of infant formula: recommendations of an ESPGHAN coordinated international expert group. J Pediatr Gastroenterol Nutr. 2005; 41 (5): 584-99.

[17] Meihong Xu, Yi Ma, Linlin Xu, Xu Y, Li Y. Multigeneration assessment of dietary nucleotides consumption in waened rats. Birth Defects Res B Dev Reprod Toxicol. 2012; 95 (6): 460-6.

[18] Reeves PG, Nielsen FH, Fahey GC: AIN-93 purified diets for laboratory rodents: final report of the American Institute of Nutrition ad hoc writing committee on the reformulation of the AIN-76A rodent diet. J Nutr. 1993; 123:1939-51.

[19] Brown EG, Newton RP, Shaw NM. Analysis of the free nucleotide pools of mammalian tissues by high pressure liquid chromatography. Anal Biochem. 1982; 123 (2): 378-88.

[20] Sheng SH, Zhang XM, Feng YQ, Wei XB, Wang SM, Hou ZL. Studies on the safety of nucleic acid oral solution. Chinese Journal of Biochemical Pharmaceutics. 1998; 20: 87-89.

[21] Zhao CY, Wu ZM, You YZ, Zhang C Mutagenic effect of nucleic acid oral solution. J Health Toxicology. 2001; 15: 124-126.

[22] Meihong Xu, Yi Ma, Linlin Xu, Xu Y, Li Y. Developmental effects of dietary nucleotides in two-generation weaned rats. J Med Food. 2013; 16 (12):1146-52.

\section{Supplementary}

Table 1. Ingredient composition of the experimental diets fed to rats ${ }^{\text {a }}$

\begin{tabular}{ccccccc}
\hline Components & Control & $0.01 \%$ NTs & $0.04 \%$ NTs & $0.16 \%$ NTs & $0.64 \%$ NTs & $1.28 \%$ NTs \\
\hline Cornstarch & & & & g/kg diet & & \\
Casein ( $\geq 85 \%$ protein) & 397.486 & 397.446 & 397.327 & 396.850 & 394.942 & 392.398 \\
Dextrinized cornstarch (90-94\% tetrasaccharides) & 200.000 & 199.980 & 199.920 & 199.680 & 198.720 & 197.440 \\
Sucrose & 132.000 & 131.987 & 131.947 & 131.789 & 131.155 & 130.310 \\
Soybean oil (no additives) & 100.000 & 99.990 & 99.960 & 99.840 & 99.360 & 98.720 \\
Fiber & 70.000 & 69.993 & 69.972 & 69.888 & 69.552 & 69.104 \\
Mineral mixture & 50.000 & 49.995 & 49.980 & 49.920 & 49.680 & 49.360 \\
Vitamin mixture & 35.000 & 34.997 & 34.986 & 34.944 & 34.776 & 34.552 \\
L-Cystein & 10.000 & 9.999 & 9.996 & 9.984 & 9.936 & 9.872 \\
ChHQ & 3.000 & 3.000 & 2.999 & 2.995 & 2.981 & 2.962 \\
Tholine bitartrate (41.1\% choline) & 2.500 & 2.500 & 2.499 & 2.496 & 2.484 & 2.468 \\
Nucleotides mixture c & 0.014 & 0.014 & 0.014 & 0.014 & 0.014 & 0.014 \\
\hline
\end{tabular}

a Ingredient compositon was based on AIN-93G rodent diet [18]

b TBHQ, tert-butylhydroquinone

c Composition of Nucleotides mixture: 22.8\%AMP, 26.6\% CMP, 30.2\% GMP (2Na), 20.4\% UMP (2Na). The purity of each of nucleotides was more than $99 \%$. 